\title{
On simple Shamsuddin derivations in two variables
}

\author{
RENE BALTAZAR \\ Instituto de Matematica, Estatística e Física, Universidade Federal do Rio Grande/FURG, \\ Santo Antônio da Patrulha, Rua Barão do Caí, 125, 95500-000 Rio Grande, RS, Brazil \\ Manuscript received on January 26, 2015; accepted for publication on September 29, 2015
}

\begin{abstract}
We study the subgroup of $k$-automorphisms of $k[x, y]$ which commute with a simple derivation $d$ of $k[x, y]$. We prove, for instance, that this subgroup is trivial when $d$ is a shamsuddin simple derivation. in the general case of simple derivations, we obtain properties for the elements of this subgroup.
\end{abstract}

Key words: dynamical degree, isotropy group, Shamsuddin derivations, simple derivations.

\section{1 - INTRODUCTION}

Let $k$ be an algebraically closed field of zero characteristic and $k[x, y]$ be the ring of polynomials over $k$ in two variables.

A $k$-derivation $d: k[x, y] \rightarrow k[x, y]$ of $k[x, y]$ is a $k$-linear map such that

$$
d(a b)=d(a) b+a d(b),
$$

for any $a, b \in k[x, y]$. We denote by $\operatorname{Der}_{\mathrm{k}}(k[x, y])$ the set of all $k$-derivations of $k[x, y]$. Let $d \in$ $\operatorname{Der}_{\mathrm{k}}(k[x, y])$. An ideal $I$ of $k[x, y]$ is called $d$-stable if $d(I) \subset I$. For example, the ideals 0 and $k[x, y]$ are always $d$-stable. If these are the only $d$-stable ideals, we say $k[x, y]$ is $d$-simple. Even in the case of two variable polynomials, only a few examples of simple derivations are known (see, for instance, Brumatti et al. 2003, Saraiva 2012, Nowicki 2008, Baltazar and Pan 2015, Kour and Maloo 2013, Lequain 2011).

We denote by $\operatorname{Aut}(k[x, y])$ the group of $k$-automorphisms of $k[x, y]$. Let $\operatorname{Aut}(k[x, y])$ act on $\operatorname{Der}_{\mathrm{k}}(k[x, y])$ by:

$$
(\rho, D) \mapsto \rho^{-1} \circ D \circ \rho=\rho^{-1} D \rho .
$$

Fix a derivation $d \in \operatorname{Der}_{\mathbf{k}}(k[x, y])$. The isotropy subgroup, with respect to this group action, is defined as

$$
\operatorname{Aut}(k[x, y])_{d}:=\{\rho \in \operatorname{Aut}(k[x, y]) / \rho d=d \rho\} .
$$

E-mail: rene.baltazar@ufrgs.br 
We are interested in the following question proposed by I.Pan (see Baltazar 2014):

Conjecture 1. If $d$ is a simple derivation of $k[x, y]$, then $\operatorname{Aut}(k[x, y])_{d}$ is finite.

Initially, in Section 2, we prove Theorem 6, which shows that the conjecture is true for a family of derivations, namely Shamsuddin derivations. For this purpose, we use a theorem due to Shamsuddin (1977) (see also Nowicki 1994, Theorem 13.2.1.) that gives a necessary and sufficient condition for a derivation to be extended to $R[t]$, with $t$ an indeterminate, and preserving simplicity. We observe Shamsuddin derivations is a reasonable class of objects. For instance, they have been previously used by Lequain (2011) in order to establish a conjecture about the Weyl algebra $\mathbb{A}_{n}$ over $k$.

In Section 3, to understand the isotropy of a simple derivation of $k[x, y]$, we give necessary conditions for an automorphism to belong to the isotropy of a simple derivation. We prove in Proposition 7 that if such an automorphism has a fixed point, then it is the identity. Next, we present the definition of dynamical degree of a polynomial map and prove in Corollary 9 that for $k=\mathbb{C}$, the elements of Aut $(\mathbb{C}[x, y])_{d}$, with $d$ a simple derivation, have dynamical degree 1 . More precisely, the condition that the dynamical degree is greater than 1 corresponds to exponential growth of the degree under iteration, and this may be viewed as a complexity of the automorphism in the isotropy (see Friedland and Milnor 1989).

\section{2 - SHAMSUDDIN DERIVATIONS}

The aim of this section is study the isotropy group of a Shamsuddin derivation in $k[x, y]$. In (Nowicki 1994, $\S 13.3)$, there are numerous examples of these derivations and a criterion for determining the simplicity. Furthermore, Lequain (2008) introduced an algorithm for determining whether a Shamsuddin derivation is simple. We begin with an example that shows that the isotropy of an arbitrary derivation can be quite complicated.

Example 1. Let $d=\partial_{x} \in \operatorname{Der}_{\mathrm{k}}(k[x, y])$ and $\rho \in \operatorname{Aut}(k[x, y])_{d}$. Note that $d$ is not a simple derivation. Indeed, for any $u(y) \in k[y]$, the ideal generated by $u(x)$ is always invariant. Consider

$$
\begin{aligned}
& \rho(x)=f(x, y)=a_{0}(x)+a_{1}(x) y+\ldots+a_{t}(x) y^{t} \\
& \rho(y)=g(x, y)=b_{0}(x)+b_{1}(x) y+\ldots+b_{s}(x) y^{s} .
\end{aligned}
$$

Since $\rho \in \operatorname{Aut}(k[x, y])_{d}$, we obtain two conditions:

1) $\rho(d(x))=d(\rho(x))$.

Thus,

$$
1=d\left(a_{0}(x)+a_{1}(x) y+\ldots+a_{t}(x) y^{t}\right)=d\left(a_{0}(x)\right)+d\left(a_{1}(x)\right) y+\ldots+d\left(a_{t}(x)\right) y^{t} .
$$

Then, $d\left(a_{0}(x)\right)=1$ and $d\left(a_{j}(x)\right)=0, j=1, \ldots, t$. We conclude that $\rho(x)$ is of the type

$$
\rho(x)=x+c_{0}+c_{1} y+\ldots+c_{t} y^{t}, \quad c_{i} \in k .
$$

2) $\rho(d(y))=d(\rho(y))$.

Analogously,

$$
0=d\left(b_{0}(x)+b_{1}(x) y+\ldots+b_{s}(x) y^{s}\right)=d\left(b_{0}(x)\right)+d\left(b_{1}(x)\right) y+\ldots+d\left(b_{s}(x)\right) y^{s},
$$


that is, $b_{i}(x)=d_{i}$, with $d_{i} \in k$. We also infer that $\rho(y)$ is of the type

$$
\rho(y)=d_{0}+d_{1} y+\ldots+d_{s} y^{s}, \quad d_{i} \in k .
$$

Thus, $\operatorname{Aut}(k[x, y])_{d}$ contains the affine automorphisms

$$
(x+u y+r, u y+s)
$$

with $u, r, s \in k$. In particular, the isotropy group $\operatorname{Aut}(k[x, y])_{d}$ of a derivation which is not simple can be infinite. Indeed, $\operatorname{Aut}(k[x, y])_{d}$ contains all automorphisms of the type $(x+p(y), y)$, with $p(y) \in k[y]$. Actually, these are all the elements of $\operatorname{Aut}(k[x, y])_{d}$. By conditions 1 and 2 ,

$$
\rho=(x+p(y), q(y))
$$

with $p(y), q(y) \in k[y]$. Since $\rho$ is an automorphism, the determinant of the Jacobian matrix must be nonzero. Thus, $\left|J_{\rho}\right|=q^{\prime}(y)=c, c \in k^{*}$. Therefore, $\rho=(x+p(y), a y+c)$, with $p(y) \in k[y]$ and $a, b \in k$. Consequently, $\operatorname{Aut}(k[x, y])_{d}$ is not finite and its first component has elements with any degree.

The following is a well known lemma.

Lemma 2. Let $R$ be a commutative ring, $d$ a derivation of $R$, and $h(t) \in R[t]$, with $t$ an indeterminate. Then, we can also extend d to a unique derivation $\tilde{d}$ of $R[t]$ such that $\tilde{d}(t)=h(t)$.

We also use the following result of Shamsuddin (1977).

Theorem 3. Let $R$ be a ring containing $\mathbb{Q}$ and let $d$ be a simple derivation of $R$. Extend the derivation $d$ to a derivation $\tilde{d}$ of the polynomial ring $R[t]$ by setting $\tilde{d}(t)=a t+b$ where $a, b \in R$. Then the following two conditions are equivalent:

(1) $\tilde{d}$ is a simple derivation.

(2) There exist no elements $r \in R$ such that $d(r)=a r+b$.

Proof. See (Nowicki 1994, Theorem 13.2.1.) for a detailed proof.

A derivation $d$ of $k[x, y]$ is said to be a Shamsuddin derivation if $d$ is of the form

$$
d=\partial_{x}+(a(x) y+b(x)) \partial_{y}
$$

where $a(x), b(x) \in k[x]$.

Example 4. Let $d$ be a derivation of $k[x, y]$ as follows

$$
d=\partial_{x}+(x y+1) \partial_{y}
$$

Writing $R=k[x]$, we know that $R$ is $\partial_{x}$-simple and, taking $a=x$ and $b=1$, we are exactly in the conditions of Theorem 3. Thus, we know that $d$ is simple if, and only if, there exist no elements $r \in R$ such that $\partial_{x}(r)=x r+1$; but the right hand side of the equivalence is satisfied by the degree of $r$. Therefore, by Theorem $3, d$ is a simple derivation of $k[x, y]$.

Lemma 5. (Nowicki 1994, Proposition. 13.3.2) Let $d=\partial_{x}+(a(x) y+b(x)) \partial_{y}$ be a Shamsuddin derivation, where $a(x), b(x) \in k[x]$. Thus, if $d$ is a simple derivation, then $a(x) \neq 0$ and $b(x) \neq 0$. 
Proof. If $b(x)=0$, then the ideal $(y)$ is $d$-invariante. If $a(x)=0$, let $h(x) \in k[x]$ such that $h^{\prime}=b(x)$, then the ideal $(y-h)$ is $d$-invariante.

One can determine the simplicity of the a Shamsuddin derivation according the polynomials $a(x)$ and $b(x)$ (see Nowicki 1994, §13.3).

Theorem 6. Let $D \in \operatorname{Der}_{\mathrm{k}}(k[x, y])$ be a Shamsuddin derivation. If $D$ is a simple derivation, then $\operatorname{Aut}(k[x, y])_{D}=\{i d\}$.

Proof. Let us denote $\rho(x)=f(x, y)$ and $\rho(y)=g(x, y)$. Let $D$ be a Shamsuddin derivation and

$$
\begin{gathered}
D(x)=1, \\
D(y)=a(x) y+b(x),
\end{gathered}
$$

where $a(x), b(x) \in k[x]$. Since $\rho \in \operatorname{Aut}(k[x, y])_{D}$, we obtain two conditions:

(1) $\rho(D(x))=D(\rho(x))$,

(2) $\rho(D(y))=D(\rho(y))$.

Then, by condition $(1), D(f(x, y))=1$ and since $f(x, y)$ can be written in the form

$$
f(x, y)=a_{0}(x)+a_{1}(x) y+\ldots+a_{s}(x) y^{s},
$$

with $s \geq 0$, we obtain

$$
\begin{gathered}
D\left(a_{0}(x)\right)+D\left(a_{1}(x)\right) y+a_{1}(x)(a(x) y+b(x))+\ldots \\
\quad+D\left(a_{s}(x)\right) y^{s}+s a_{s}(x) y^{s-1}(a(x) y+b(x))=1 .
\end{gathered}
$$

By comparing the coefficients of $y^{s}$,

$$
D\left(a_{s}(x)\right)=-s a_{s}(x) a(x)
$$

which can not occur by simplicity. More explicitly, Lemma 5 implies $a(x)=0$. Thus, $s=0$, that is, $f(x, y)=a_{0}(x)$. Therefore, $D\left(a_{0}(x)\right)=1$ and $f=x+c$, with $c$ constant.

By using condition (2),

$$
\begin{aligned}
D(g(x, y)) & =\rho(a(x) y+b(x)) \\
& =\rho(a(x)) \rho(y)+\rho(b(x)) \\
& =a(x+c) g(x, y)+b(x+c) .
\end{aligned}
$$

By the previous part, we can suppose that $t>0$, because $\rho$ is a automorphism. Now, write $g(x, y)=$ $b_{0}(x)+b_{1}(x) y+\ldots+b_{t}(x) y^{t}$. Thus,

$$
\begin{aligned}
a(x+c) g(x, y)+b(x+c)= & D\left(b_{0}(x)\right)+D\left(b_{1}(x)\right) y+b_{1}(x)(a(x) y+b(x))+ \\
& +\ldots+D\left(b_{t}(x)\right) y^{t}+t b_{t}(x) y^{t-1}(a(x) y+b(x)) .
\end{aligned}
$$


By comparing the coefficients of $y^{t}$, we obtain

$$
D\left(b_{t}(x)\right)+t b_{t}(x) a(x)=a(x+c) b_{t}(x) .
$$

Then $D\left(b_{t}(x)\right)=b_{t}(x)(-t a(x)+a(x+c))$. In this way, $b_{t}(x)$ is a constant and, consequently, $a(x+c)=$ $t a(x)$. Comparing the coefficients in the last equality, we obtain $t=1$ and then $b_{1}(x)=b_{1}$ is constant. Moreover, if $a(x)$ is not a constant, since $a(x+c)=a(x)$, it is easy to see that $c=0$. Indeed, if $c \neq 0$, we obtain that the polynomial $a(x)$ has infinite distinct roots. If $a(x)$ is constant, then $a(x) \cdot D$ is not a simple derivation (this is a consequence of Lequain 2008, Lemma.2.6 and Theorem.3.2); thus, we obtain $c=0$. Note that $g(x, y)=b_{0}(x)+b_{1} y$ and, using the condition (2),

$$
\begin{array}{r}
D(g(x, y))=\quad D\left(b_{0}(x)\right)+b_{1}(a(x) y+b(x)) \\
=a(x)\left(b_{0}(x)+b_{1} y\right)+b(x) .
\end{array}
$$

Considering the independent term of $y$, we have

$$
D\left(b_{0}(x)\right)=b_{0}(x) a(x)+b(x)\left(1-b_{1}\right) .
$$

By (Nowicki 1994, Proposition. 13.3.3), if $b_{1} \neq 1$, we have that $D$ is a simple derivation if and only if $D^{\prime}$, defined by

$$
D^{\prime}(x)=1, \quad D^{\prime}(y)=a(x) y+b(x)\left(1-b_{1}\right),
$$

is a simple derivation. Furthermore, by Theorem 3, there exist no elements $h(x)$ in $K[x]$ such that

$$
D(h(x))=h(x) a(x)+b(x)\left(1-b_{1}\right) .
$$

This contradicts equation (1). Then, $b_{1}=1$ and $D\left(b_{0}(x)\right)=b_{0}(x) a(x)$. Since $D$ is a simple derivation, we know that $a(x) \neq 0$ and consequently $b_{0}(x)=0$. This shows that $\rho=i d$.

\section{3 - ON THE ISOTROPY OF THE SIMPLE DERIVATIONS}

The purpose of this section is to study the isotropy in the general case of a simple derivation. More precisely, we obtain results that reveal nice features of the elements of $\operatorname{Aut}(k[x, y])_{D}$. For this, we use some concepts presented in the previous sections and the concept of dynamical degree of a polynomial map.

In Baltazar and Pan (2015), which was inspired by Brumatti et al. (2003), the authors introduce and study a general notion of solution associated to a Noetherian differential $k$-algebra and its relationship with simplicity.

The following proposition has a geometrical flavour: it says that if an element in the isotropy of a simple derivation has fixed point, then it is the identity automorphism.

Proposition 7. Let $D \in \operatorname{Der}_{\mathrm{k}}\left(k\left[x_{1}, \ldots, x_{n}\right]\right)$ be a simple derivation and $\rho \in \operatorname{Aut}\left(k\left[x_{1}, \ldots, x_{n}\right]\right)_{D}$ be an automorphism in the isotropy. Suppose that there exists a maximal ideal $\mathfrak{m} \subset k\left[x_{1}, \ldots, x_{n}\right]$ such that $\rho(\mathfrak{m})=$ $\mathfrak{m}$, then $\rho=i d$. 
Proof. Let $\varphi$ be a solution of $D$ passing through $\mathfrak{m}$ (see (Baltazar and Pan 2015, Definition.1.)). We know that $\frac{\partial}{\partial t} \varphi=\varphi D$ and $\varphi^{-1}((t))=\mathfrak{m}$. If $\rho \in \operatorname{Aut}\left(k\left[x_{1}, \ldots, x_{n}\right]\right)_{D}$, then

$$
\frac{\partial}{\partial t} \varphi \rho=\varphi D \rho=\varphi \rho D
$$

In other words, $\varphi \rho$ is a solution of $D$ passing through $\rho^{-1}(\mathfrak{m})=\mathfrak{m}$. Then, by the uniqueness of the solution (Baltazar and Pan 2015, Theorem.7.(c)), $\varphi \rho=\varphi$. Because $k\left[x_{1}, \ldots, x_{n}\right]$ is $D$-simple and $\varphi$ is a nontrivial solution, we have that $\varphi$ is one-to-one. Therefore, $\rho=i d$.

Lane (1975) proved that every $k$-automorphism $\rho$ of $k[x, y]$ leaves a nontrivial proper ideal $I$ invariant over an algebraically closed field, that is, $\rho(I) \subseteq I$. In Shamsuddin (1982), Shamsuddin proved that this result does not extend to $k[x, y, z]$, proving that the $k$-automorphism given by $\chi(x)=x+1, \chi(y)=$ $y+x z+1$, and $\chi(z)=y+(x+1) z$ has no nontrivial invariant ideal.

In addition, since $k[x, y]$ is Noetherian, $\rho$ leaves a nontrivial proper ideal $I$ invariant if, and only if, $\rho(I)=I$. In fact, the ascending chain

$$
I \subset \rho^{-1}(I) \subset \rho^{-2}(I) \subset \ldots \subset \rho^{-l}(I) \subset \ldots
$$

must stabilize; thus, there exists a positive integer $n$ such that $\rho^{-n}(I)=\rho^{-n-1}(I)$. Hence, $\rho(I)=I$.

Suppose that $\rho \in \operatorname{Aut}(k[x, y])_{D}$ and that $D$ is a simple derivation of $k[x, y]$. By Proposition 7, if this invariant ideal $I$ is maximal, we have $\rho=i d$. Suppose that $I$ is radical and let $I=\left(\mathfrak{m}_{1} \cap \ldots \cap \mathfrak{m}_{s}\right) \cap\left(\mathfrak{p}_{1} \cap\right.$ $\left.\ldots \cap \mathfrak{p}_{t}\right)$ be a primary decomposition, where the ideals $\mathfrak{m}_{i}$ are maximal and $\mathfrak{p}_{j}$ are prime ideals with height 1 such that $\mathfrak{p}_{j}=\left(f_{j}\right)$, with $f_{j}$ irreducible (see Kaplansky 1974, Theorem 5). If

$$
\rho\left(\mathfrak{m}_{1} \cap \ldots \cap \mathfrak{m}_{s}\right)=\mathfrak{m}_{1} \cap \ldots \cap \mathfrak{m}_{s}
$$

we claim that $\rho^{N}$ leaves invariant one maximal ideal for some $N \in \mathbb{N}$. Indeed, we know that $\rho\left(\mathfrak{m}_{1}\right) \supset$ $\mathfrak{m}_{1} \cap \ldots \cap \mathfrak{m}_{s}$ and since $\rho\left(\mathfrak{m}_{1}\right)$ is a prime ideal, we deduce that $\rho\left(\mathfrak{m}_{1}\right) \supseteq \mathfrak{m}_{i}$, for some $i=1, \ldots, s$ (Atiyah and Macdonald 1969, Prop.11.1.(ii)). Then, $\rho\left(\mathfrak{m}_{1}\right)=\mathfrak{m}_{i}$, that is, $\rho^{N}$ leaves invariant the maximal ideal $\mathfrak{m}_{1}$, for some $N \in \mathbb{N}$. Thus, it follows from Proposition 7 that $\rho^{N}=i d$.

Note that $\rho\left(\mathfrak{p}_{1} \cap \ldots \cap \mathfrak{p}_{t}\right)=\mathfrak{p}_{1} \cap \ldots \cap \mathfrak{p}_{t}$. In fact, writing $\mathfrak{p}_{1} \cap \ldots \cap \mathfrak{p}_{t}=\left(f_{1} \ldots f_{t}\right)$, with $f_{i}$ irreducible, we would like to choose $h \in \mathfrak{m}_{1} \cap \ldots \cap \mathfrak{m}_{s}$ such that $\rho(h) \notin \mathfrak{p}_{1}$. If such $h$ does not exist, we would obtain $\mathfrak{m}_{1} \cap \ldots \cap \mathfrak{m}_{s} \subset \mathfrak{p}_{1}$, then $\mathfrak{p}_{1} \supseteq \mathfrak{m}_{i}$, for some $i=1, \ldots, s$ (Atiyah and Macdonald 1969, Prop.11.1.(ii)): a contradiction. Thus, since $h f_{1} \ldots f_{t} \in I$, we obtain $\rho(h) \rho\left(f_{1}\right) \ldots \rho\left(f_{t}\right) \in I \subset \mathfrak{p}_{1}$. Therefore, $\rho\left(f_{1} \ldots f_{t}\right) \in$ $\mathfrak{p}_{1}$. Likewise, the same conclusion holds for the other prime ideals $\mathfrak{p}_{i}, i=1, \ldots, t$. Finally, $\rho\left(\mathfrak{p}_{1} \cap \ldots \cap \mathfrak{p}_{t}\right)=$ $\mathfrak{p}_{1} \cap \ldots \cap \mathfrak{p}_{t}$.

In the next corollary, we obtain consequences on the case of radical ideals.

Corollary 8. Let $\rho \in \operatorname{Aut}(k[x, y])_{D}, D$ a simple derivation of $k[x, y]$, and $I=(f)$ an ideal with height 1 such that $\rho(I)=I$, with $f$ reduced. If $V(f)$ is singular or some irreducible component $C_{i}$ of $V(f)$ has genus greater than two, then $\rho$ is an automorphism of finite order.

Proof. Suppose that $V(f)$ is not a smooth variety and let $q$ be a singularity of $V(f)$. Since the set of singular points is invariant by $\rho$, there exists $N \in \mathbb{N}$ such that $\rho^{N}(q)=q$. Using that $\rho \in \operatorname{Aut}(k[x, y])_{D}$, we obtain, by Proposition $7, \rho^{N}=i d$. 
Let $C_{i}$ be a component irreducible of $V(f)$ that has genus greater than two. Note that there exists $M \in \mathbb{N}$ such that $\rho^{M}\left(C_{i}\right)=C_{i}$. By (Farkas and Kra 1992, Theorem Hunvitz, p.241), the number of elements in $\operatorname{Aut}\left(C_{i}\right)$ is finite; in fact, $\#\left(\operatorname{Aut}\left(C_{i}\right)\right)<84\left(g_{i}-1\right)$, where $g_{i}$ is the genus of $C_{i}$. Then, we deduce that $\rho$ is an automorphism of finite order.

In the rest of this section, we let $k=\mathbb{C}$.

Consider a polynomial map $f(x, y)=\left(f_{1}(x, y), f_{2}(x, y)\right): \mathbb{C}^{2} \rightarrow \mathbb{C}^{2}$ and define the degree of $f$ by $\operatorname{deg}(f):=\max \left(\operatorname{deg}\left(f_{1}\right), \operatorname{deg}\left(f_{2}\right)\right)$. Thus, we may define the dynamical degree (see Blanc and Deserti (in press), Friedland and Milnor 1989, Silverman 2012) of $f$ as

$$
\delta(f):=\lim _{n \rightarrow \infty}\left(\operatorname{deg}\left(f^{n}\right)\right)^{\frac{1}{n}} .
$$

Corollary 9. If $\rho \in \operatorname{Aut}(\mathbb{C}[x, y])_{D}$ and $D$ is a simple derivation of $\mathbb{C}[x, y]$, then $\delta(\rho)=1$.

Proof. Suppose $\delta(\rho)>1$. By (Friedland and Milnor 1989, Theorem 3.1.), $\rho^{n}$ has exactly $\delta(\rho)^{n}$ fixed points counted with multiplicities. Then, by Proposition 7, $\rho=i d$, which shows that the dynamical degree of $\rho$ is 1 .

\section{ACKNOWLEDGMENTS}

I would like to thank Ivan Pan for his comments and suggestions. Research of R. Baltazar was partially supported by Coordenação de Aperfeiçoamento de Pessoal de Nível Superior (CAPES).

\section{REFERENCES}

ATIYAH MF AND MACDONALD IG. 1969. Introduction to Commutative Algebra. Massachusetts: Addison-Wesley Publishing Company.

BALTAZAR R. 2014. Sobre soluções de derivações em k-algebras Noetherianas e simplicidade. Tese de Doutorado, Universidade Federal do Rio Grande do Sul.

BALTAZAR R AND PAN I. 2015. On solutions for derivations of a Noetherian k-algebra and local simplicity. Commun Algebra 43(7): 2739-2747.

BLANC J AND DESERTI J. IN PRESS. Degree Growth of Birational Maps of the Plane.

BRUMATTI P, LEQUAIN Y AND LEVCOVITZ D. 2003. Differential simplicity in Polynomial Rings and Algebraic Independence of Power Series. J London Math Soc 68(3): 615-630.

FARKAS HM AND KRA I. 1992. Riemann Surfaces. 2nd edition. Graduate Texts in Mathematics, Springer.

FRIEDLAND S AND MILNOR J. 1989. Dynamical properties of plane polynomial automorphisms. Ergodic Theory Dyn Syst 9: 67-99.

KAPLANSKY I. 1974. Commutative Rings. Chicago, (2nd edition).

KOUR S AND MALOO AK. 2013. Simplicity of Some Derivations of k[x, y]. Commun Algebra 41(4): 1417-1431.

LANE DR. 1975. Fixed points of affine Cremona transformations of the plane over an algebraically closed field. Amer J Math 97(3): 707-732.

LEQUAIN Y. 2008. Simple Shamsuddin derivations of K[X; Y1; :.:; Yn]: An algorithmic characterizarion. J Pure Appl Algebra 212(4): 801-807.

LEQUAIN Y. 2011. Cyclic irreducible non-holonomic modules over the Weyl algebra: An algorithmic characterization. J Pure Appl Algebra 215(4): 531-545.

NOWICKI A. 1994. Polynomial derivations and their rings of constants. TORUN. at http://www-users.mat.umk.pl/anow/psdvi/pol-der.pdf.

NOWICKI A. 2008. An Example of a Simple Derivation in Two Variables. Colloq Math 113(1): 25-31.

SARAIVA C. 2012. Sobre Derivações Simples e Folheações holomorfas sem Solução Algébrica, Tese de Doutorado. 
SHAMSUDDIN A. 1977. Automorphisms and Skew Polynomial Rings. Ph.D. thesis, Univesity of Leeds.

SHAMSUDDIN A. 1982. Rings with automorphisms leaving no nontrivial proper ideals invariant. Canadian Math Bull 25: $478-486$.

SILVERMAN JH. 2012. Dynamical Degrees, Arithmetic Degrees, and Canonical Heights for Dominant Rational Self-Maps of

Projective Space. Ergodic Theory and Dynamical Systems 34(2): 647-678. 